Revue des patrimoines

\title{
La bourrine : architecture rurale en bauge et couverture végétale dans le Nord-Ouest de la Vendée
}

David Milcent

\section{OpenEdition}

Journals

Édition électronique

URL : http://journals.openedition.org/insitu/2977

DOI : 10.4000/insitu.2977

ISSN : 1630-7305

Éditeur

Ministère de la culture

Référence électronique

David Milcent, «La bourrine : architecture rurale en bauge et couverture végétale dans le Nord-Ouest de la Vendée », In Situ [En ligne], 7 | 2006, mis en ligne le 18 avril 2012, consulté le 03 mai 2019. URL http://journals.openedition.org/insitu/2977; DOI : 10.4000/insitu.2977

Ce document a été généré automatiquement le 3 mai 2019.

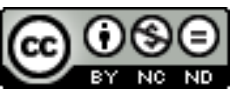

In Situ Revues des patrimoines est mis à disposition selon les termes de la licence Creative Commons Attribution - Pas d'Utilisation Commerciale - Pas de Modification 4.0 International. 


\title{
La bourrine : architecture rurale en bauge et couverture végétale dans le Nord-Ouest de la Vendée
}

\author{
David Milcent
}

1 Maison bâtie en terre et couverte en roseaux, la bourrine du marais de Monts $^{1}$, en Vendée, constitue un exemple remarquable d'une utilisation optimale des ressources naturelles d'une région à des fins constructives. Faisant appel, dans sa mise en œuvre, à des techniques issues de pratiques anciennes ${ }^{2}$, la bourrine étonne par l'ingéniosité qu'ont su développer ses constructeurs pour s'adapter à un milieu hostile. Habiter dans le marais de Monts revenait à subir les intempéries hivernales et les inondations qui, jusque dans la seconde moitié du XX siècle, isolaient le marais durant plusieurs mois (fig. $\left.\mathbf{n}^{\circ} \mathbf{1}\right)$. 


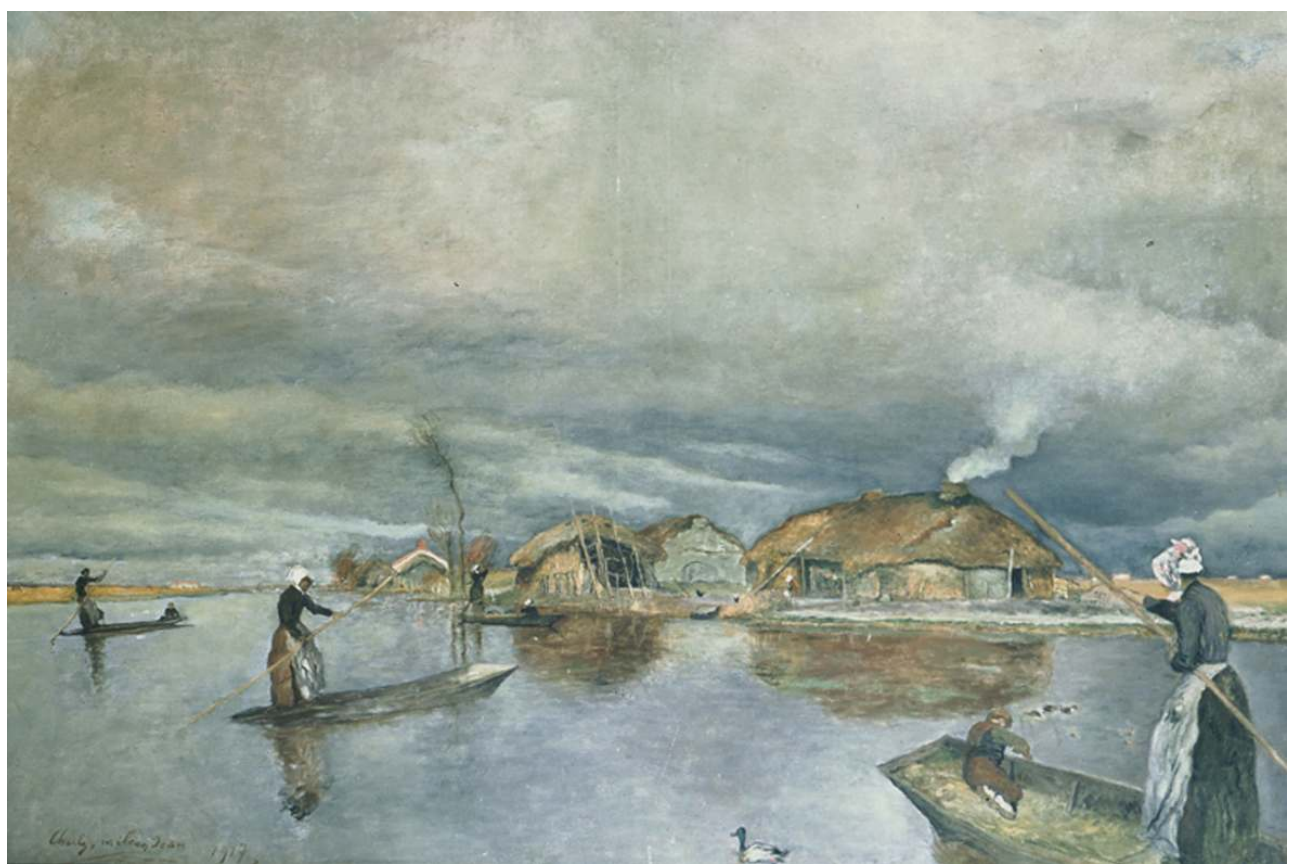

Avant la grêle, huile sur toile, Charles Milcendeau, 1917

(c) Musée Vendéen de Fontenay-le-Comte

\section{La bauge dans le marais de Monts : la technique du bigôt}

On ne peut évoquer la construction des bourrines sans porter un regard sur leurs couvertures végétales, auxquelles renvoie le terme ${ }^{3}$. Cependant l'examen des murs en terre crue révèle un mode constructif original dans le marais de Monts qui serait à rapprocher des techniques de construction en bauge observées, entre autres, dans la région de Rennes et dans les marais du Cotentin et du Bessin ${ }^{4}$.

Depuis le XVII ${ }^{e}$ siècle, le terme bauge est utilisé pour décrire une technique spécifique de construction en terre qui est distincte de celle du pisé, de l'adobe et du torchis ${ }^{5}$. A l'examen des constructions présentes dans l'ouest de la France et des mentions dans la littérature ${ }^{6}$, on peut définir la bauge ${ }^{7}$ comme une technique d'apprêt de la terre crue qui, mélangée à de l'eau et des fibres végétales, acquiert d'indéniables qualités plastiques; sa mise en œuvre s'effectue par assises successives sans l'aide de coffrage ni d'aucun autre support. La technique dite du bigôt, utilisée dans le marais de Monts, illustre parfaitement cette pratique (fig. $\mathbf{n}^{\circ} \mathbf{2}$ ). 


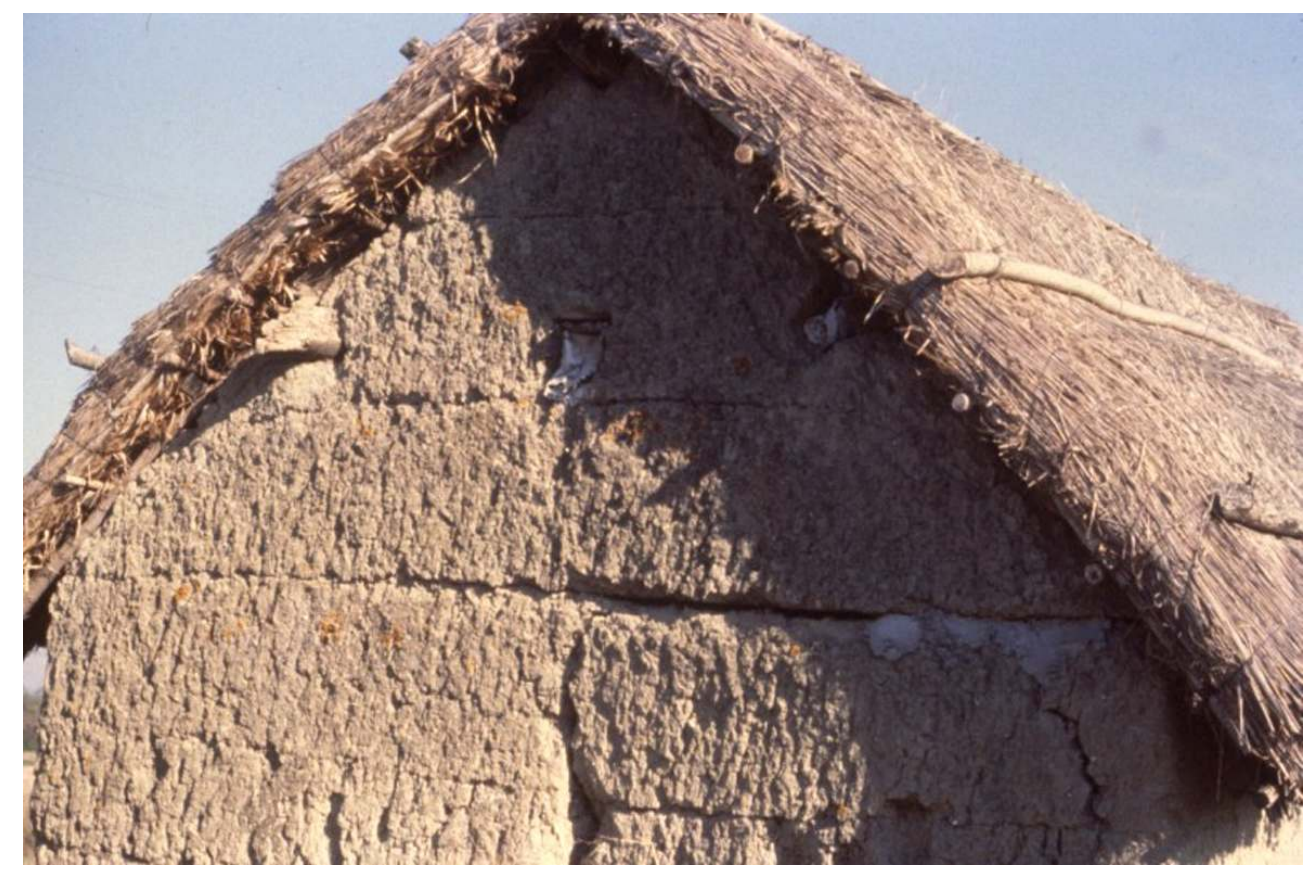

Mur en bigôts, v. 1980

(C) Conservation Départementale des Musées de Vendée

\section{La mise en œuvre}

4 Prélevée sous la couche de terre arable, la terre ${ }^{8}$ à construire est disposée en tas à l'emplacement même du chantier, à proximité d'un fossé pour les besoins d'approvisionnement en eau. Mais également, «dans bien des cas, les bâtisseurs récupéraient les matériaux d'une bourrine effondrée, tant la bauge que les pièces de charpente $»^{9}$. Une fois détrempée, la terre est foulée aux pieds par les hommes euxmêmes ou par des animaux (bovins), puis retournée régulièrement à l'aide d'une fourche ou d'une fraï ${ }^{10}$ jusqu'à obtenir une matière homogène. Au cours de cette opération, l'ajout de sable prélevé dans les dunes ${ }^{11}$ avoisinantes permet d'alléger la terre, réduisant ainsi le retrait provoqué au séchage. La préparation obtenue doit être suffisamment souple pour y inclure des fibres végétales (foin, paille et rouche $e^{12}$ ), destinées à armer et stabiliser le mélange. Cette dernière opération permet alors de façonner, à l'aide de la fourche à bigôter ${ }^{13}$, des moellons de terre, les bigôts, éléments de base servant à édifier les murs. Ces derniers sont alors assemblés en assises, les levées, qui mesurent au plus cinquante centimètres de haut et environ soixante centimètres de large. La première assise était généralement disposée à même le sol. L'excédent de terre provoqué par le tassement des levées était récupéré à l'aide de la fourche et placé au sommet du mur à la fin de la journée de travail ${ }^{14}$.

5 Après quelques jours, nécessaires au séchage, les constructeurs préparaient à nouveau la terre afin de répéter l'opération. Les gouttereaux sont ainsi élevés jusqu'à une hauteur approximative d'un mètre quatre-vingts, tandis que pignons et murs de refends peuvent atteindre une hauteur de quatre mètres. 
6 Les encadrements des portes et fenêtres sont posés au fur et à mesure que s'élèvent les murs, dans lesquels on effectuait des réserves comprenant appuis et linteaux. La pièce commune recevait généralement deux portes pleines et une fenêtre à quatre carreaux. Les huisseries étaient simplement montées sur pivots.

7 La famille et les proches participent aux travaux, tandis qu'une seule personne est chargée d'élever les murs. Une journée de travail suffit pour mettre en forme une levée qui concerne l'ensemble des murs du bâtiment - gouttereaux, pignons et murs de refends - de manière à assurer une meilleure cohésion entre eux. La construction des murs s'étalait ainsi durant un mois; généralement entrepris au printemps, elle bénéficie de conditions climatiques optimales pour le séchage de la terre.

\section{Les solins}

8 Les murs des bourrines sont pour la plupart posés à même le sol, sans fondations ni soubassements. Parfois les constructeurs creusent une tranchée d'une vingtaine de centimètres dans laquelle ils placent la première levée de bigôts.

Dans les bourrines élevées aux abords de secteurs plus riches en pierre, on a su tirer parti de cette proximité. On observe en effet, sur quelques maisons et dépendances, des solins appareillés en moellons de calcaire ou en galets provenant de la plage. Ces derniers dépassent rarement $40 \mathrm{~cm}$ de hauteur, mais ils permettent sans aucun doute d'isoler le mur en bauge des remontées, par capillarité, de l'humidité du sol si préjudiciable aux murs en terre.

10 Dans les dépendances, appelées localement galeries, de quelques fermes importantes, la bauge est utilisée comme élément de remplissage entre des piliers en moellons de pierre qui supportent la charpente.

\section{La protection des murs}

11 Il est nécessaire de protéger les murs du vent et de la pluie, dont les effets d'érosion sont accentués du fait de l'absence d'arbres dans certaines parties du marais de Monts. Aussi a$\mathrm{t}$-on pris autrefois l'habitude de placer des claies de roseau ${ }^{15}$, les fretis, sur les parois les plus exposées. On applique aussi un enduit de terre et de sable sur les faces intérieure et extérieure des murs recevant un chaulage. Bien que pratiqué depuis fort longtemps à l'intérieur de la pièce habitable, le chaulage des murs extérieurs semble n'avoir été pratiqué que depuis le début du siècle ${ }^{16}\left(\right.$ fig. $\mathbf{n}^{\circ}$ 3). 
Figure 3

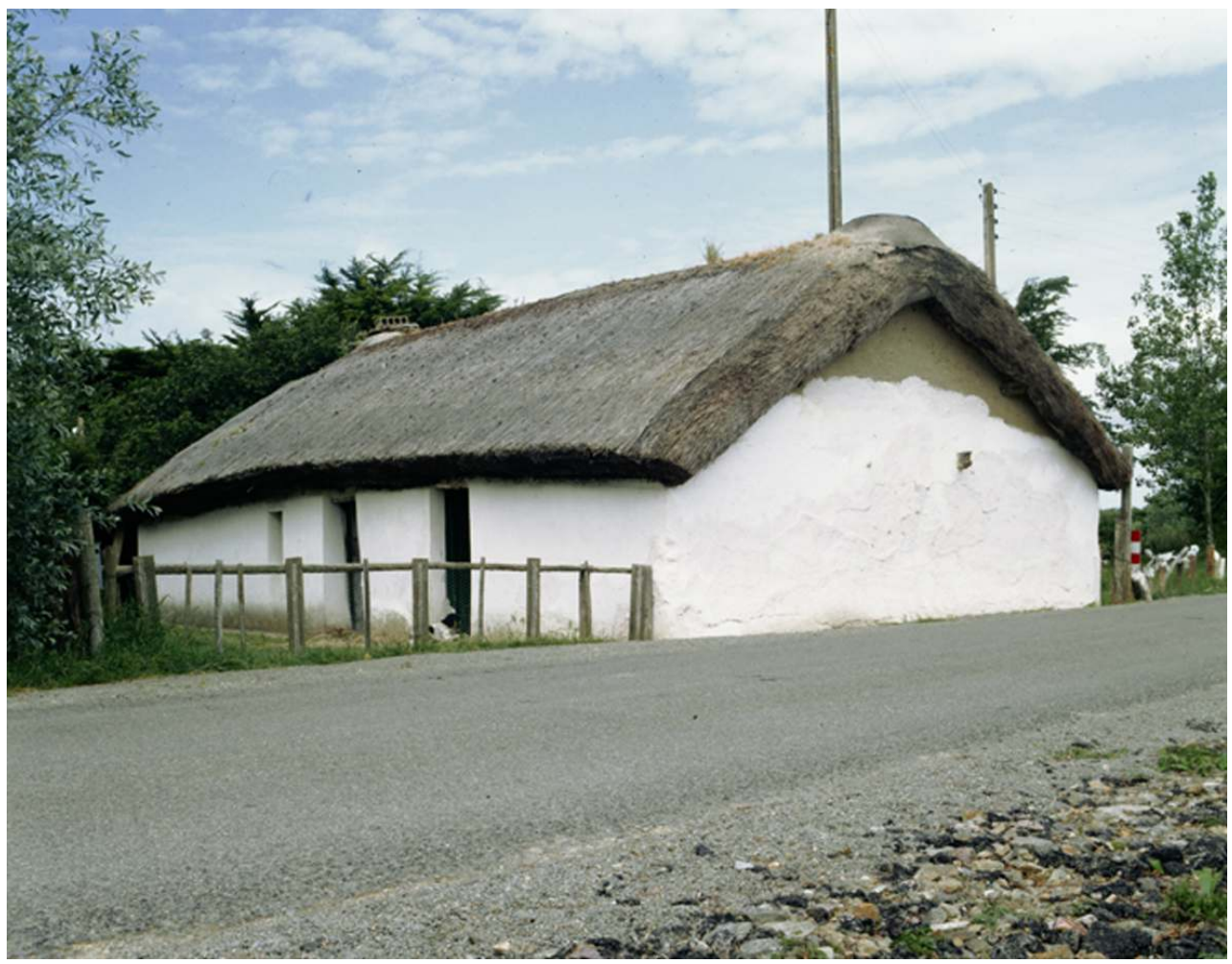

Les Charbonnières, Notre-Dame-de-Monts

Phot. Inv. B. Renoux @ Inventaire Général, ADAGP, 2002

Bien que plus rare dans le marais de Monts, l'enduit de chaux s'observe également sur certaines parois de terre. Afin d'en faciliter l'adhérence, des galets ou des morceaux de tuiles sont placés au préalable dans le mur.

\section{La cheminée et le four à pain}

Elément indispensable de la pièce habitable, la cheminée est, elle aussi, construite en terre. Adossée à un des murs-pignons, la hotte est faite d'un mélange de terre et de foin appliqué sur une armature en branches. L'ensemble est recouvert d'un enduit et chaulé tout comme les murs de la pièce (fig. $\mathbf{n}^{\circ} \mathbf{4}$ ). 
Figure 4

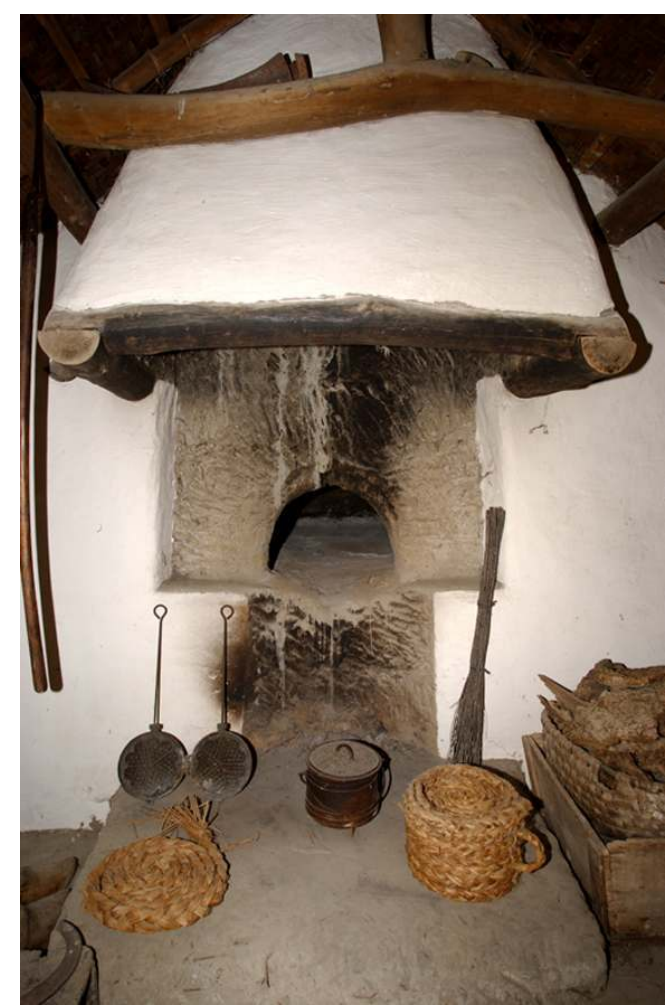

Cheminée et four à pain, bourrine à Chartier. Ecomusée du marais Breton Vendéen - Le Daviaud (c) Conservation Départementale des Musées de Vendée, 2004

Le four comprend une masse, construite en bigôts, qui supporte la sole, elle-même couverte par la voûte. La sole, initialement en bauge, fut progressivement remplacée par des carreaux de terre cuite, moins fragiles. La voûte est constituée de bigôts de terre et foin, façonnés à la main sur un moule en sable. Son extrados est protégé par un toit de roseau que porte un mur de bauge ou de simples poteaux de bois. Le four à pain, adossé au mur-pignon sur lequel s'élève la cheminée, est accessible depuis la pièce habitable.

\section{La charpente}

Le bois étant quasi inexistant dans le marais, les éléments de charpente proviennent le plus souvent de remplois divers ou de bosquets d'ormes, de saules et de peupliers. Plus tard, la forêt de Monts, plantée au milieu du XIX ${ }^{\mathrm{e}}$ siècle sur les dunes des communes littorales, permit un approvisionnement plus aisé en bois (pin maritime).

16 La charpente est posée sur les murs en terre qui en supportaient tout le poids. Les constructeurs n'utilisent pas de sablières censées en répartir les charges. Les chevrons étaient pris dans la bauge lors d'une opération de remplissage entre murs et charpente appelée arasure. Seule la pièce la plus importante, la ferme, repose parfois sur un poteau de bois placé dans la maçonnerie lors de l'édification des murs. Observé dans les ruines ou lors des démolitions, ce poteau n'a été repéré que dans les pièces d'habitation et semble être inexistant dans les dépendances. 
Figure 5

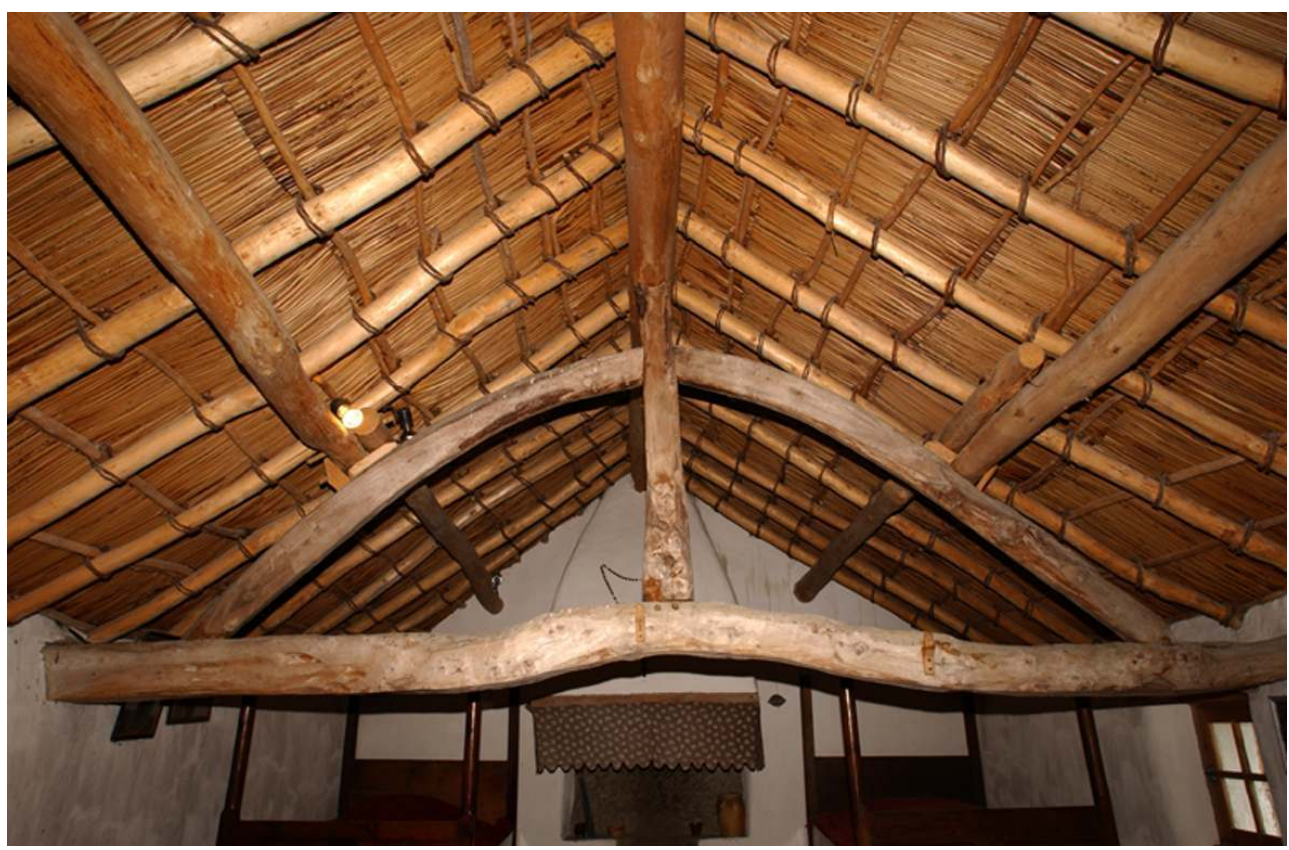

Charpente, bourrine à Chartier. Ecomusée du marais Breton Vendéen - Le Daviaud

Phot. P. Durandet (C) Conservation Départementale des Musées de Vendée, 2004

L'entrait, généralement incurvé, facilite le passage des habitants au centre de la pièce (fig. $\mathbf{n}^{\circ}$ 5). Le tirant ${ }^{17}$, les arbalétriers également courbes dans la plupart des cas observés et le poinçon sont assemblés de manière rudimentaire à tenons et mortaises. Cette opération est généralement confiée à un charpentier. Les pannes, chevrons et liteaux, simples branches et troncs écorcés, sont assemblés par le propriétaire qui liait ces pièces grâce à des liens de bois (saules ou jeunes pousses d'orme). Une croupe placée sur le pignon ouest offre une meilleure résistance aux intempéries et permet le plus souvent d'organiser l'étable autour d'un poteau central qui, en forme de fourche dans sa partie supérieure, reçoit la panne faîtière (fig. $\mathbf{n}^{\circ} \mathbf{6}$ ). 
Figure 6

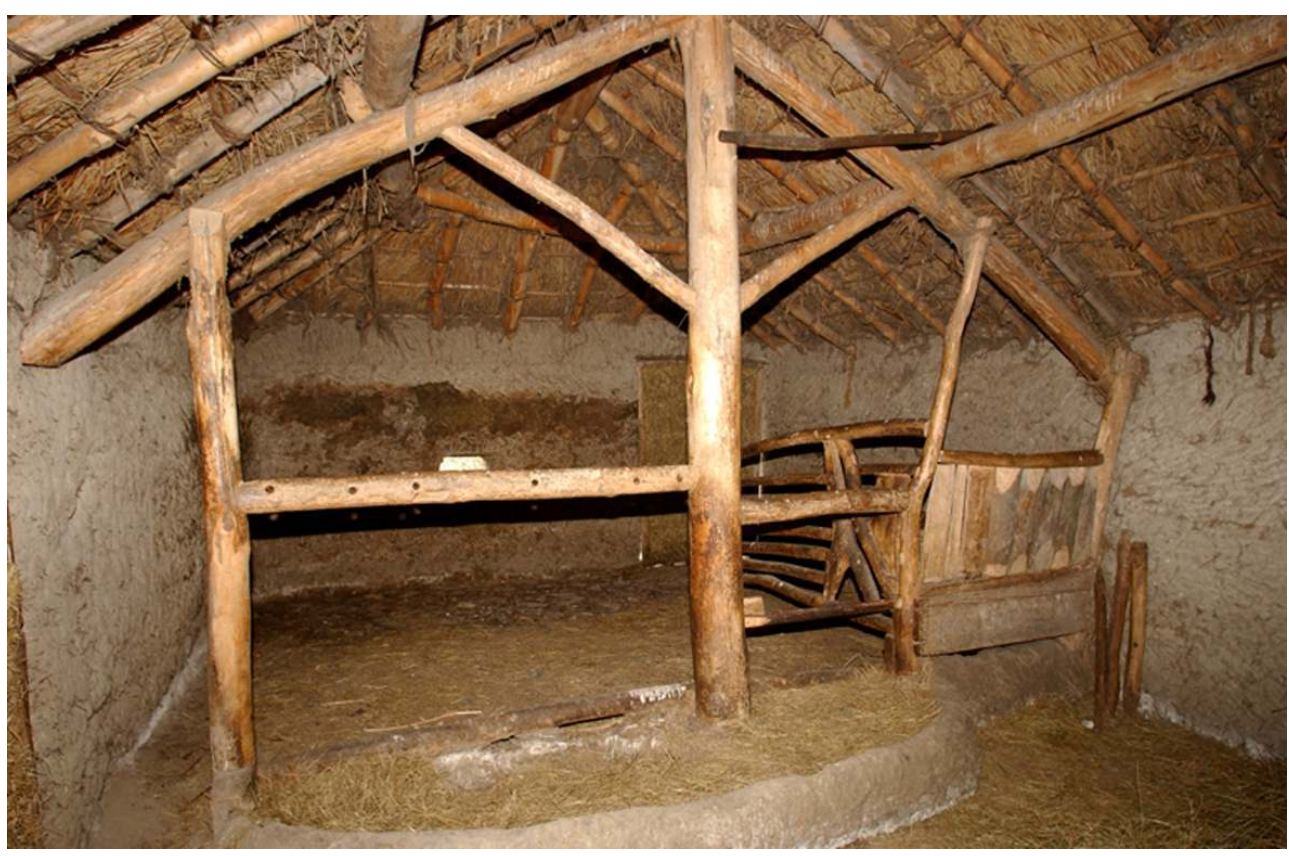

Grange-étable, bourrine à Chartier. Ecomusée du marais Breton Vendéen - Le Daviaud

Phot. P. Durandet (C) Conservation Départementale des Musées de Vendée, 2004

\section{La couverture végétale}

Souvent interdit à cause des risques d'incendie en milieu urbain et freiné par la mécanisation de l'agriculture, l'usage du chaume ${ }^{18}$ a progressivement diminué. Considéré comme « la couverture la plus courante depuis les Gaulois jusqu'à la fin du XVIII ${ }^{\mathrm{e}}$ siècle » ${ }^{19}$, le chaume a perduré dans les zones de marais et marécages où l'exploitation de la matière première a été possible plus longtemps qu'ailleurs : la Grande Brière, la Camargue et le marais de Monts ${ }^{20}$.

\section{Le matériau}

Les espèces végétales utilisées dans le marais de Monts pour couvrir les bourrines diffèrent selon la localisation de la construction. On utilisera volontiers des végétaux tels que le phragmite et la massette dans le marais doux, le scirpe maritime dans le marais saumâtre.

20 Le roseau ou phragmite commun (Phragmites australis) se récolte sur les bords des canaux et dans les roselières du marais doux. Sa tige, terminée par une panicule, est utilisée pour sa rigidité et sa longueur - de 1 à 3 mètres. Il est présent dans la majorité des couvertures des bourrines du marais de Monts.

21 La massette (Typha angustifolia) est reconnaissable par sa fleur appelée quenouille. Utilisée en couverture dans le marais de Bouin ${ }^{21}$, elle sert également de sous-couche dans les différentes dépendances du marais de Monts. Ses feuilles auraient servi à confectionner des parures dans certaines dépendances. 

dans d'anciens marais salants exploités en roselières appelés rouchères. De section triangulaire, il peut atteindre 1,2 mètre de longueur. Il était utilisé pour couvrir les bourrines des communes de Beauvoir-sur-Mer et La Barre-de-Monts ainsi que certaines bourrines situées à Notre-Dame-de-Monts.

Le scirpe d'eau douce ou jonc des chaisiers (Scirpus lacustris), appelé localement rouche ou rouche ronde, peut atteindre 3 mètres de longueur. Sa tige ronde, lisse et spongieuse, permet de fabriquer nombre d'objets du quotidien des habitants du marais. Il permet de confectionner une parure esthétique en sous-toiture et pouvait servir comme matériau de couverture $^{22}$.

\section{La coupe}

24

La période de la coupe diffère quelque peu en fonction des espèces. Cependant, elle a lieu après la période estivale et avant les pluies automnales. Seul le scirpe lacustre est coupé avant l'été.

Prélevés dans des roselières, d'anciens marais salants ou sur les bords des principaux canaux et fossés, ces végétaux sont récoltés à l'aide d'une faucille, puis nettoyés et posés au sol en javelles pour former gerbes ou menoilles ${ }^{23}$. Il convient par la suite de les mettre en gerbes et de les assembler en piles (fig. $\mathbf{n}^{\circ} \mathbf{7}$ ), sortes de meules coniques destinées au stockage, ou bien de les acheminer à proximité du chantier, assemblées en mouches. Récolté le plus souvent par l'habitant lui-même, le roseau peut également faire l'objet d'un commerce. 


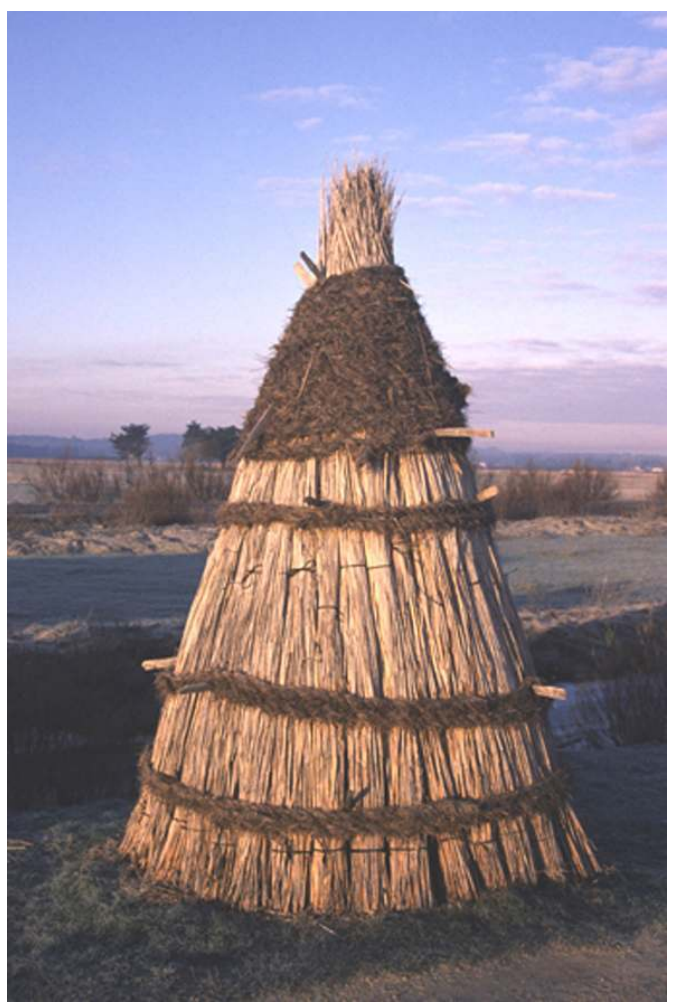

Pile de roseaux

Phot. D. Milcent @ Ecomusée du marais Breton Vendéen - Le Daviaud, 2000

\section{Le savoir-faire}

La pose de la couverture des bourrines est confiée à un spécialiste (fig. $\left.\mathbf{n}^{\circ} \mathbf{8}\right)$. D'abord reconnu pour son savoir-faire, le bourrinour est devenu à partir du début du $\mathrm{XX}^{\mathrm{e}}$ siècle un artisan spécialisé. Jean Bossu, lors de l'enquête sur l'architecture rurale, fait une description détaillée de l'activité d'un bourrinour dans une monographie de $1943^{24}: \mathrm{M}$. Joseph Pineau, à Saint-Jean de Monts, offre ses services aux habitants du voisinage pour les différents travaux de couverture. Travaillant seul, il œuvre à partir du mois d'octobre jusqu'au printemps, le commanditaire fournissant la matière première. Ce dernier peut également participer aux travaux en procédant à l'approvisionnement du chantier. Le savoir-faire nécessaire à la bonne exécution de ce mode de couverture se reflète dans la spécificité de l'outillage qui «se compose d'une échelle d'environ 3 mètres de long, de la palette, du couteau, de sa pierre à aiguiser, de l'aiguille, d'une paire de tenailles et d'un baquet de chevilles" 


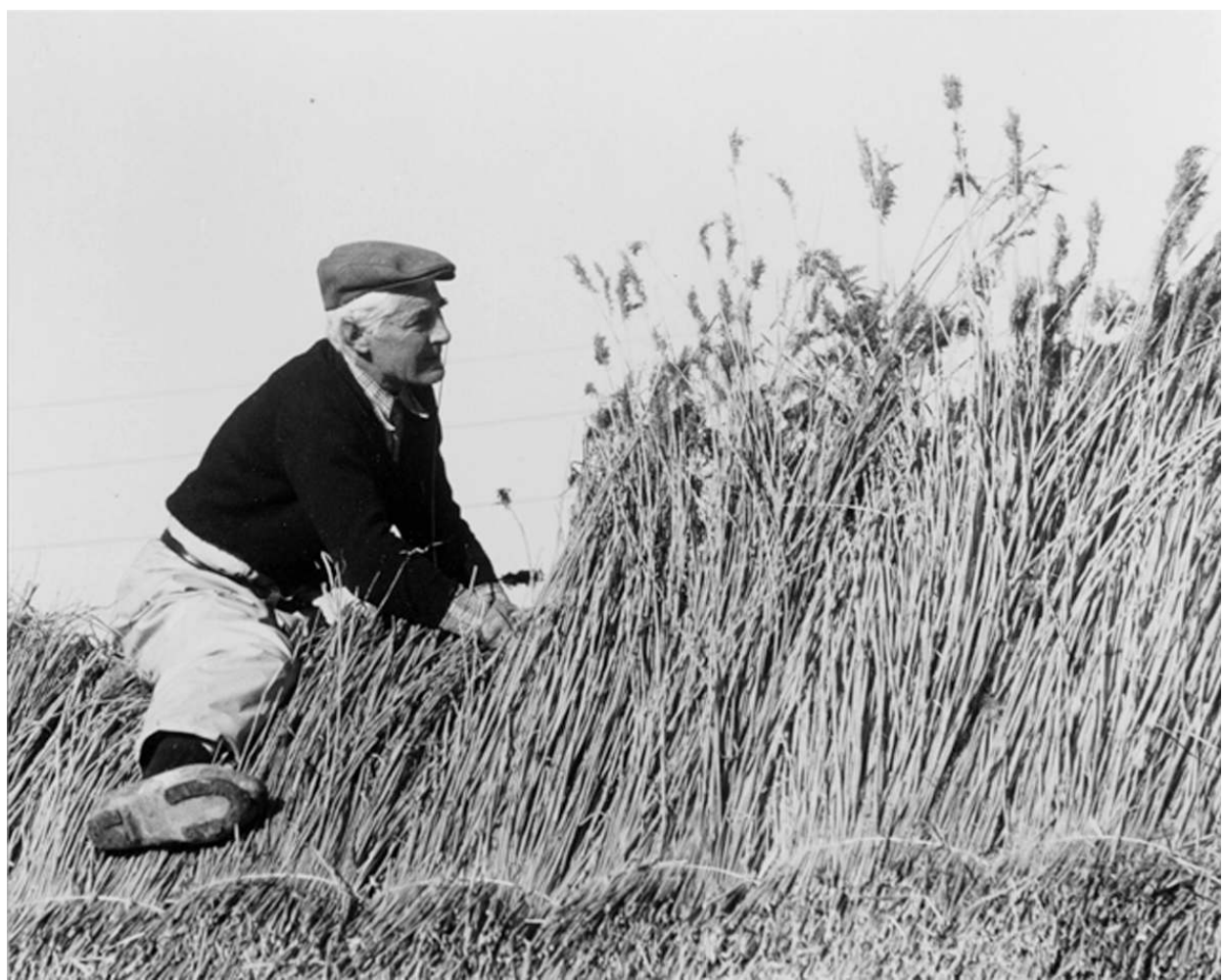

Raymond Maréchal, bourrinour, restauration d'une bourrine à Notre-Dame-de-Monts Collection Particulière

\section{La pose de la couverture}

Débordant de 15 centimètres environ du mur de bauge, le chevron permet de fixer sur un liteau placé à l'extérieur du mur une menoille liée qui enfourche ce dernier. Ce montage est appelé la goubleture. La saillie ainsi obtenue en posant les premiers roseaux sur la goubleture, est d'environ vingt centimètres, ce qui permettait de diriger le ruissellement loin du pied de mur.

Dans les pièces d'habitation, qui ne sont pas couvertes par un plafond, la face inférieure de la couverture, appelée sous-couche, est visible et doit par conséquent présenter un aspect propre et soigné. Le scirpe lacustre (jonc des chaisiers) et la paille de seigle ${ }^{25}$ permettent de réaliser une parure soignée du plus bel aspect. Dans quelques bourrines, on trouve également une natte (fig. $\mathbf{n}^{\circ}$ 9) de végétaux tissés qui offre une meilleure longévité. 
Figure 9

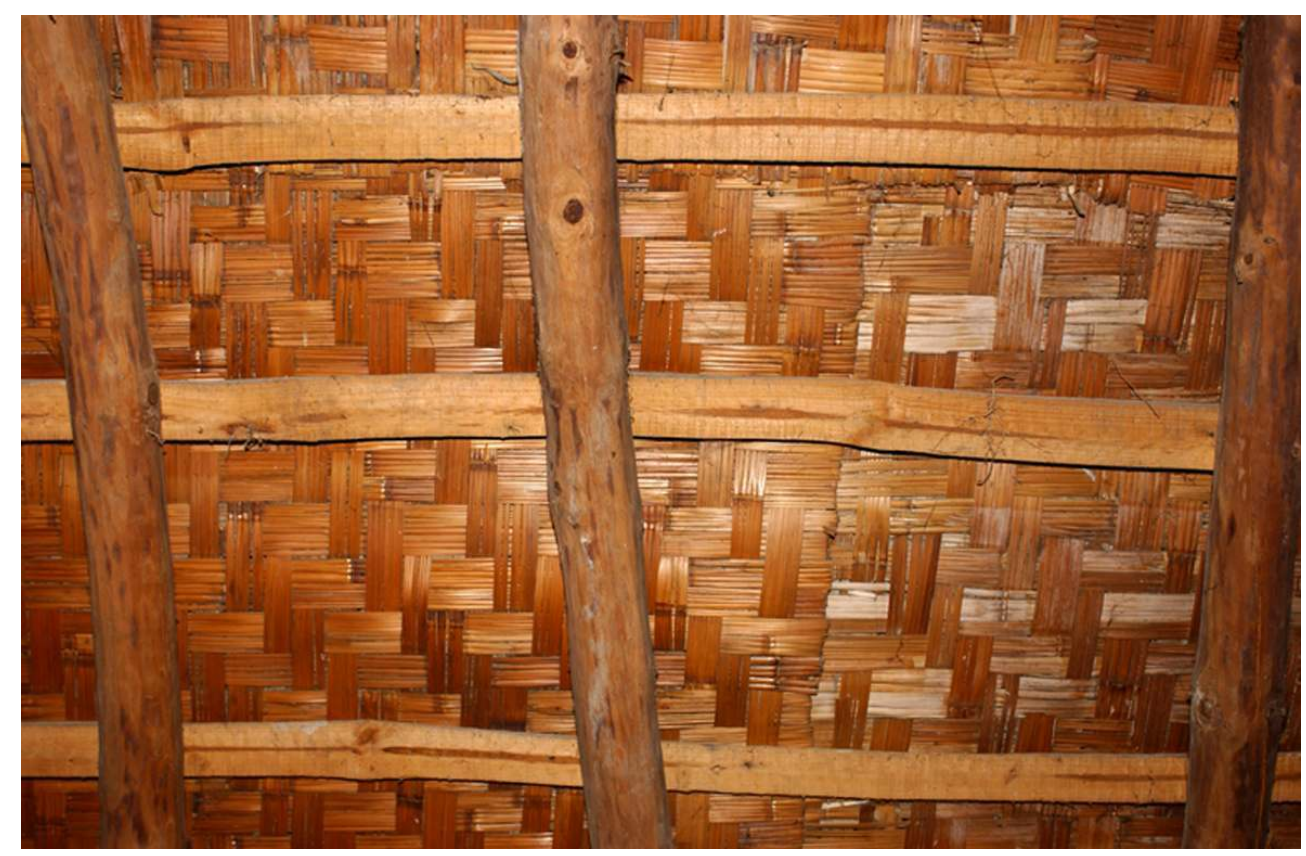

Rive maintenue par des liens végétaux

Phot. D. Milcent @ Ecomusée du marais Breton Vendéen - Le Daviaud, 2000

La pose des roseaux, qui prennent appui sur la goubleture et la parure, débute à hauteur de l'égout du mur-pignon et se poursuit de manière horizontale, par passées. L'épaisseur moyenne est d'environ trente-cinq centimètres. Le bourrinour étale soigneusement les roseaux puis, à l'aide d'une palette, confère à la pente du toit - environ $45^{\circ}$ - une surface régulière et homogène. Des liens végétaux servent à fixer les bottes de roseaux (massette et phragmite) sur la charpente, contrairement aux couvertures en scirpe maritime qui ne sont pas liées ${ }^{26}$. Depuis 1920, l'emploi du fil de fer a fait disparaître les liens végétaux au profit de ce matériau solide et imputrescible. La passée horizontale effectuée, le bourrinour harmonisait la surface à l'aide de la palette mais aussi avec un couteau ou rasoir (extrémité d'une lame de faux munie d'un manche).

30 Les rives sont exécutées en même temps que les passées horizontales du rampant. Les roseaux du versant sont préalablement bloqués par des chevilles de bois traversant la couverture. Un bourrelet sert de forme pour créer l'arrondi de la rive. Fabriqué avec de la rouche maintenue avec des liens de foin, il est fixé sur le mur pignon par des chevilles de bois. Cette rive sera maintenue par des liens végétaux dont « on [...] tirait [...] un effet décoratif ${ }^{27}$ (fig. $\mathbf{n}^{\circ}$ 10). 
Figure 10

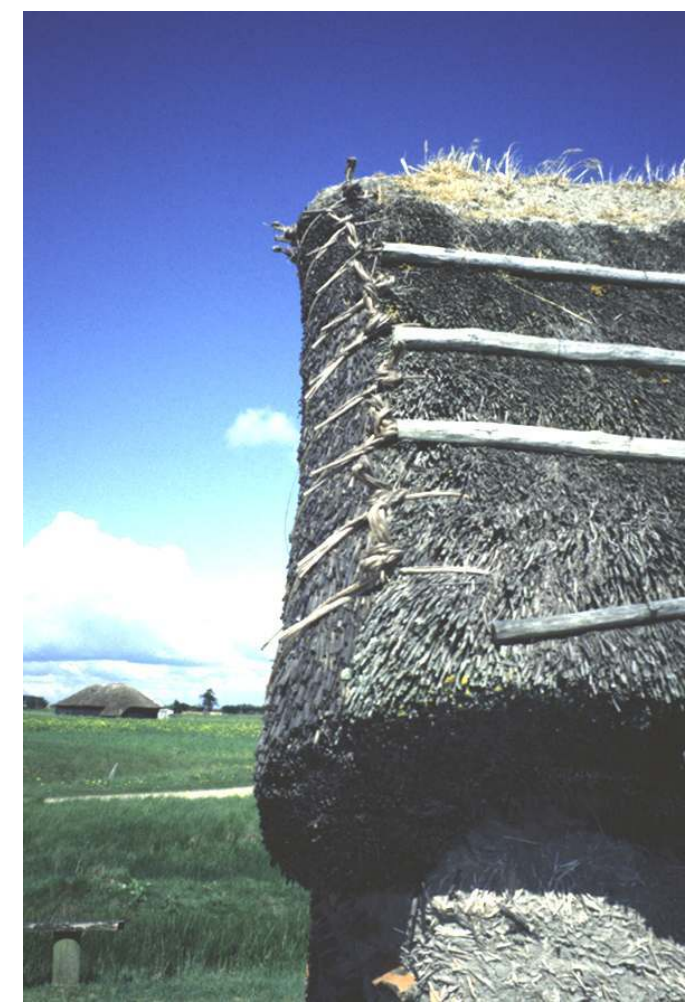

Natte

Phot. P. Durandet (C) Conservation Départementale des Musées de Vendée, 2004

Constitué par un enchevêtrement des roseaux qui se trouvent sur la partie supérieure des deux pans de la toiture, le faitage est recouvert par un mortier de terre qui protège ce point faible. Ce dernier est planté de joubarbes et de figuiers de Barbarie ${ }^{28}$ qui servent à le stabiliser. Les arêtiers de la croupe sont protégés par un mortier de terre qui rejoint le faîtage de même nature. Après 1950, on trouvait parfois des faîtages réalisés en mortier de chaux et « blanchis » au lait de chaux.

\section{Implantations et dépendances}

Dans le marais, la bourrine est principalement implantée le long des voies de communication et notamment sur les délaissés de charrauds ${ }^{29}$. Son constructeur veillait à choisir un endroit plus ou moins surélevé afin de limiter les inconvénients des inondations. Les Maraîchins prenaient soin d'orienter leurs maisons de manière à bénéficier de l'ensoleillement de la façade, mais aussi de limiter les dégradations occasionnées par le vent ; la disposition de la croupe généralement dirigée vers l'ouest en est un témoignage (fig. $\left.\mathbf{n}^{\circ} \mathbf{1 1}\right)$. 
Figure 11

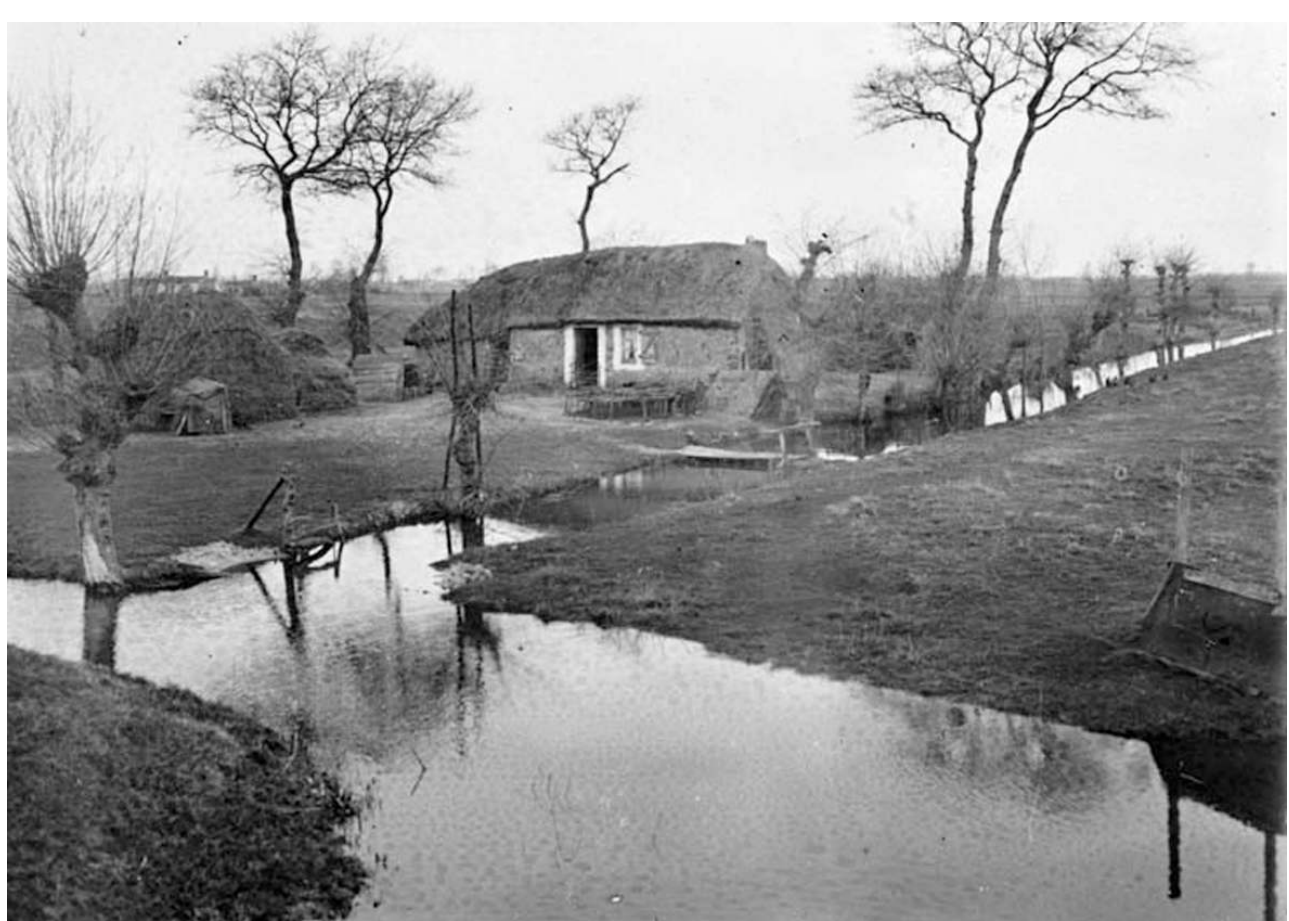

Bourrine dans le marais

Phot. J. Roussière @ Conservation Départementale des Musées de Vendée

Quelques photographies anciennes et l'examen des archives de l'Office National des Forêts attestent la présence des bourrines dans les dunes et sur ses rives. Aujourd'hui, quelques rares bourrines et dépendances en bauge témoignent encore dans ce secteur de l'occupation antérieure à la plantation de la forêt de Monts. Elles subsistent dans les "parées », enclaves forestières conservées malgré la politique d'aménagement et de fixation des dunes menée par l'Etat au milieu du XIX'e siècle.

De nombreuses dépendances d'exploitations agricoles - de métairies, de borderies ou de bourrines - étaient réalisées en terre et couvertes en roseaux afin de répondre aux besoins des exploitants. Edifiées au fur et à mesure de l'évolution de l'exploitation en s'adossant le plus souvent à un pignon, ces dépendances venaient compléter l'édifice. L'étable, la galerie ${ }^{30}$, la laiterie, la boulangerie ${ }^{31}$ et autres petits bâtiments d'élevage - le pondoir, le perchoir, le « toit » à cochons - sont implantés selon la disponibilité des lieux et leur usage (fig. $\left.\mathbf{n}^{\circ} \mathbf{1 2}\right)$. 


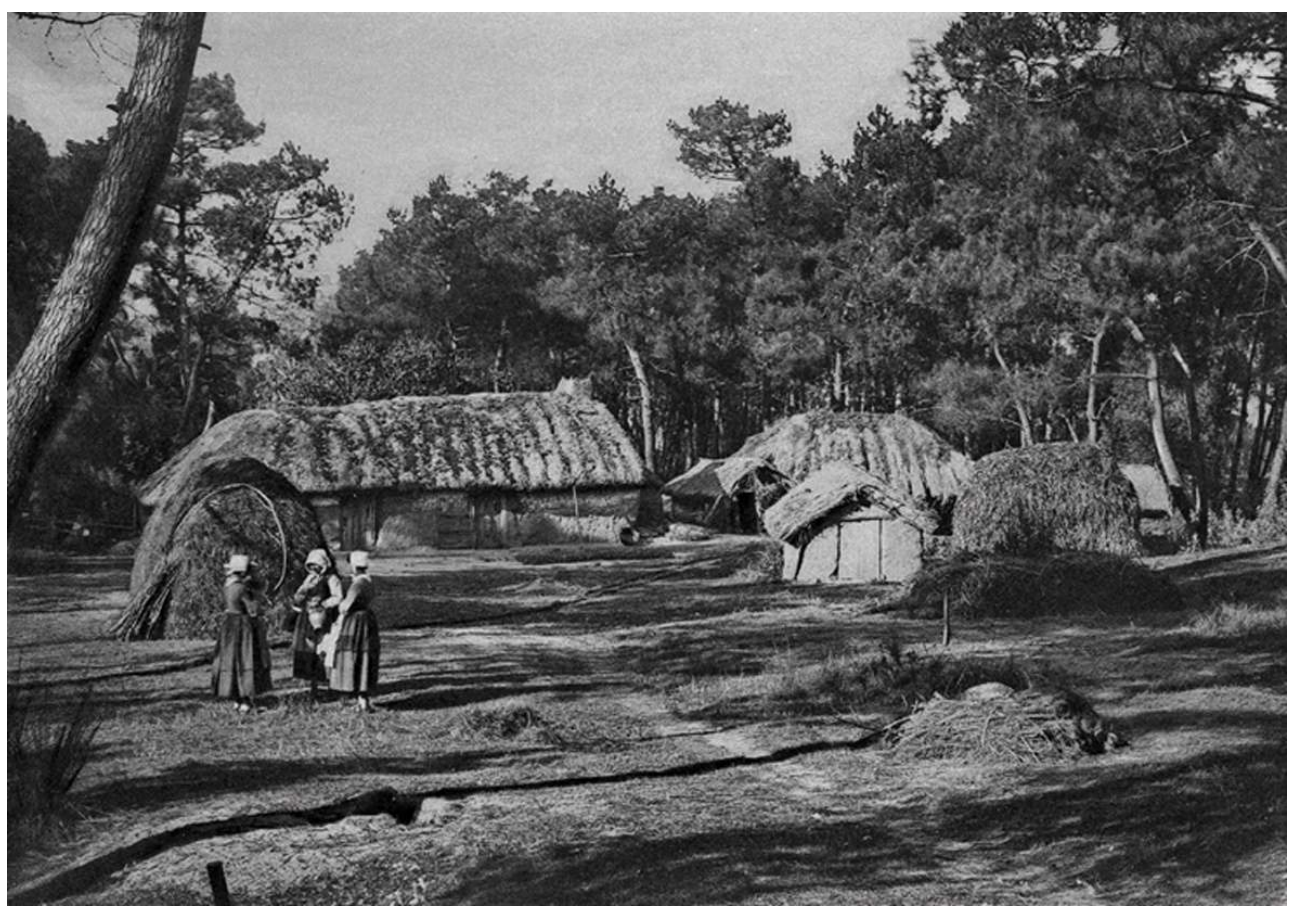

Bourrine du Pont de l'Arche, Saint-Hilaire-de-Riez, vers 1890

Phot. J.-C. Robuchon ( C Conservation Départementale des Musées de Vendée

Ainsi, édifiées par ses futurs occupants et leurs proches, les bourrines ont permis de répondre aux besoins en logement d'une population rurale généralement indigente. L'apparition de nombreuses briqueteries, tuileries et fours à chaux au XIX ${ }^{\mathrm{e}}$ siècle, ainsi que l'amélioration des voies de communication permettront aux maraîchins d'accéder à de nouveaux matériaux de construction. Puis, la généralisation de l'usage du ciment et des premiers agglomérés vers $1920^{32}$ auront raison de cette tradition constructive et de ce savoir-faire après la Seconde Guerre mondiale. Dès lors, la population du marais de Monts s'est tournée vers d'autres «modes d'habiter ", d'autant plus volontiers qu'à la bourrine était attachée une connotation négative de pauvreté et du fait également du statut précaire de ces bâtiments : élevés sur les délaissés de charrauds, ils sont implantés sur une portion du domaine public. Même s'il subsiste quelques rares bourrines et dépendances, de nombreux remaniements en gênent aujourd'hui considérablement la lecture.

\section{NOTES}

1. Nous ne connaissons pas de description ni de représentation anciennes de bourrines, dont l'existence est pourtant attestée au moins depuis le XIV ${ }^{\mathrm{e}}$ siècle. Notre étude repose sur un corpus restreint d'édifices, environ 80 bourrines et dépendances à ce jour, sur quelques références bibliographiques, ainsi que sur le témoignage contemporain de certains habitants du marais. 
Nicard, Laurence. Etude du Patrimoine du canton de Saint-Jean de Monts. Communauté de Communes Océan-Marais de Monts, Saint-Jean de Monts, septembre 2000. Mission d'Inventaire menée sur le canton de Saint-Jean de Monts par Laurence Nicard de 1997 à 2000 à la demande de la Communauté de Communes Océan-Marais de Monts et le Service régional de l'Inventaire des Pays de la Loire. Voir le site : http://www.ecomusee-ledaviaud.com/

2. Echanges transdisciplinaires sur les constructions en terre crue - Terre modelée, découpée ou coffrée ; les matériaux et modes de mise en œuvre. Actes de la table-ronde de Montpellier des 17-18 novembre 2001, dir. Claire-Anne de Chazelles et Alain Klein. Montpellier : Editions de l'Espérou, 2003, p. 275 à 331.

3. Bourrine : dérivant du latin burra, laine grossière, celle-ci renvoie à la silhouette touffue des toitures faites de roseaux assemblées en fagots. Le Bœuf, François. Maison de terre et de roseau, la bourrine du marais de Monts. Terres d'Architecture : regards sur les bourrines du marais de Monts. Communauté de Communes Océan Marais de Monts. La Mothe-Achard: Editions Offset 5, septembre 2004, p. 39.

4. Bardel, Philippe, Maillard, Jean-Luc. Construction de terre en Ille-et-Vilaine. Ecomusée du Pays de Rennes. Rennes : Editions Apogée, 2002. Streiff, François, Lahure, Franck. Le patrimoine en bauge de haute et basse Normandie. Développement actuel des savoir-faire et la filière bauge en Normandie. Echanges transdisciplinaires sur les constructions en terre crue, 1, tableronde de Montpellier des 17-18 novembre 2001. Montpellier : Editions de l'Espérou, 2003, p. 311 à 326.

5. CRATerre. Traité de construction en Terre. Marseille : Editions Parenthèses, 1989, p. 14.

6. Patte, Erwan. Entre Sèves et Taute. De terre et de pierre dans les marais du Cotentin. Direction régionale des affaires culturelles de Basse-Normandie, service régional de l'inventaire. Cabourg : Cahiers du Temps, 2004 (coll. Images du patrimoine, $n^{\circ} 232$ ).

7. Bauge : technique qui permet de modeler des formes directement sans l'aide de moulage ou de coffrage, en utilisant la plasticité des terres humides. La terre ne sert pas à remplir une armature comme le torchis, mais on la façonne directement comme une poterie. La qualité plastique du matériau est donc le facteur essentiel de la mise en cuvre. Il s'agit de trouver l'état de consistance intermédiaire entre une terre trop sèche et impossible à façonner et une boue trop humide, manquant de cohésion. CRATerre. Construire en terre. Marseille: Editions Parenthèses, 1985. Voir également: Lahure, Franck. Pisé ou bauge ? Paris : Maisons Paysannes de France, $n^{\circ} 111,1994$, p. 30.

8. Le sol du marais de Monts est constitué de bri (terre argilo-sableuse de couleur bleu-gris).

9. Bertrand, Jean-Pierre. Maisons traditionnelles du Nord-Ouest Vendéen. Saint-Jean-deMonts : Editions Mémoire des Vendéens, AREXCPO, 1995, p. 114.

10. Fraïe : pelle-bêche étroite et profilée du marais de Monts.

11. Un cordon dunaire, long d'environ 20 kilomètres, sépare le marais de Monts de l'Océan Atlantique.

12. Rouche : scirpe maritime (Bolboschoenus maritimus).

13. Fourche à bigôter: fourche en bois à trois doigts. Perraudeau, Gilles. Les bourrines du marais Nord Vendéen, témoignages d'une histoire et d'une culture. Bassac: Editions Séquences, 1988. Egalement, fourche à trois doigts appelée fourche à bigôter. Bertrand, Jean-Pierre. Maisons traditionnelles du Nord-Ouest Vendéen. Saint-Jean-de-Monts: Editions Mémoire des Vendéens, AREXCPO, 1995, p. 115.

14. Un grand nombre de dépendances qui n'étaient pour la plupart jamais enduites laissent apparaître sur les murs intérieurs en bauge les trois doigts épais de la fourche.

15. Perraudeau, Gilles. Les bourrines du marais nord-vendéen, témoins d'une histoire et d'une culture. Bassac : Editions Séquences, 1988, p. 47.

16. Perraudeau, Gilles. Les bourrines du marais nord-vendéen, témoins d'une histoire et d'une culture. Bassac: Editions Séquences, 1988, p. 46. Bertrand, Jean-Pierre. Maisons 
traditionnelles du Nord-Ouest Vendéen. Saint-Jean-de-Monts: Editions "Mémoire des Vendéens ", AREXCPO, 1995, p. 119.

17. Tirant : appellation locale désignant l'entrait.

18. Chaume: terme regroupant l'ensemble des couvertures végétales traditionnelles en genêt, roseau, jonc, bruyère, seigle et blé.

19. Lebouteux, Pierre. Traité de couverture traditionnelle. Pove del Grappa, Italie : Editions H. Vial, novembre 2001, p. 13.

20. On notera les efforts de conservation d'une tradition de chaume dans l'ensemble de ces territoires (mais également en Normandie) où faute de pouvoir s'approvisionner en seigle et roseaux de pays, on utilise du roseau de Camargue récolté industriellement.

21. La massette était récoltée dans des roselières situées entre Bouin et Machecoul, à Port-laRoche.

22. D'après une communication orale avec M. Maurice Bodin, 2004.

23. Menoille, appellation locale désignant une petite gerbe de roseau correspondant à une poignée et qui était lié par deux longueurs de scirpe "mou " qui, une fois torsadé, formait un lien robuste. Le scirpe maritime, assemblé en gerbes, devait être trié préalablement à la pose ou au stockage - on ôtait les feuilles et les graines.

24. Bossu, Jean. Enquête sur l'architecture rurale, le marais de Monts. Paris : Musée National des Arts et Traditions Populaires, 1943, monographie $n^{\circ} 5$.

25. D'après une communication orale avec M. Jean-Louis Billon, 2001. Une parure en seigle a pu être observée lors de la restauration de la couverture de la bourrine la Davière à Notre-Dame-deMonts.

26. Technique de couverture considérée comme la plus ancienne du marais de Monts, la couverture en rouche ou subaù révèle une technique originale. Disposés sans lien par passées horizontales, ces végétaux sont simplement maintenus sur la charpente par leur poids. Des garlattes, branches de peupliers ou de pins maritimes empêchent ces végétaux de se soulever sous l'action du vent et donc de déstructurer la couverture.

27. Lebouteux, Pierre. Traité de couverture traditionnelle. Pove del Grappa, Italie : Editions H. Vial, novembre 2001, p. 13.

28. On observe les figuiers de Barbarie seulement à Saint-Hilaire-de-Riez; ils sont absents du marais de Monts.

29. Délaissé de charraud : partie élargie d'un chemin. Ce délaissé était propriété de la commune ; son utilisation devait faire l'objet d'un accord préalable. L'utilisateur ne jouissait pas d'un titre de propriété, la construction d'une bourrine était simplement tolérée à cet endroit.

30. Galerie : appellation locale servant à désigner une dépendance destinée à stocker le matériel agricole. Ses murs sont en bigôts, roseaux, bois, piliers ronds ou rectangulaires en moellons; sa couverture est en chaume.

31. La boulangerie désigne le fournil.

32. Bertrand, Jean-Pierre. Maisons traditionnelles du Nord-Ouest Vendéen. Saint-Jean-deMonts : Editions Mémoire des Vendéens, AREXCPO, 1995, p. 56. 


\section{RÉSUMÉS}

La bourrine du marais de Monts en Vendée, bâtie en terre et couverte en roseaux, témoigne de l'utilisation optimale des ressources naturelles d'une région. Attestée depuis le XIV ${ }^{\mathrm{e}}$ siècle, la bourrine relève d'un mode constructif ancien. Les murs édifiés en bigôts - appellation locale désignant la bauge - et la couverture en chaume, révèlent l'adresse des maraîchins qui ont su tirer profit de la terre et des roseaux à des fins constructives. La bourrine a ainsi permis de répondre aux besoins en logement des habitants les plus modestes qui utilisaient volontiers les délaissés de charrauds, ou bords de chemin, pour y installer habitations et dépendances.

The «bourrine » is the name given to a building of earth construction with its roof thatched in reeds. It bears witness to the optimal use of the natural resources of the region where it is found, the marshes of the Monts in the Vendée. It is an ancient type of construction, attested since the 14th century. The walls were made of «bigôts », a local word for the wattle-type construction of earth and straw, and the roof was in thatching. Their builders thus made good use of the soil and the reeds of the marshes. The "bourrine" satisfied the dwelling needs of the most modest sections of society, which often installed them by the roadside where they found room for the houses and their outbuildings.

\section{INDEX}

Mots-clés : architecture rurale, bauge, bigôt, bourrine, chaume, couverture végétale, délaissé de charraud, fretis, jonc, marais, Marais de Monts, massette, Notre-Dame-de-Monts, roseaux, SaintJean-de-Monts, scirpe, terre, Vendée

Keywords : earth, earth and straw construction, Marais de Monts, reeds, rural architecture, thatch, Vendée, wattle, bauge, bourrine

\section{AUTEUR}

\section{DAVID MILCENT}

Chargé de mission patrimoine. Communauté de Communes Océan-Marais de Monts, Ecomusée du marais Breton Vendéen - Le Daviaud. david.milcent@free.fr 